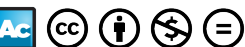

\title{
Protein-Ligand Binding Molecular Details Revealed by Terahertz Optical Kerr Spectroscopy: A Simulation Study
}

\author{
Zhijun Pan, Jing Huang,* and Wei Zhuang*
}

Cite This: JACS Au 2021, 1, 1788-1797

Read Online

ABSTRACT: Picosecond fast motions and their involvement in the biochemical processes such as protein-ligand binding has engaged significant attention. Terahertz optical Kerr spectroscopy (OKE) has the superior potential to probe these fast motions directly. Application of OKE in protein-ligand binding study is, however, limited by the difficulty of quantitative atomistic interpretation, and the calculation of Kerr spectrum for entire solvated protein complex was considered not yet feasible, due to the lack of one consistent polarizable model for both configuration sampling and polarizability calculation. Here, we analyzed the biochemical relevance of OKE to the lysozyme-triacetylchitotriose binding based on the first OKE simulation using one consistent Drude polarizable model. An analytical multipole and induced dipole scheme was employed to calculate the off-diagonal Drude polarizability more efficiently and accurately. Further theoretical analysis revealed how the subtle twisting and stiffening of aromatic protein residues' spatial arrangement as well as the confinement of small water clusters between ligand and protein cavity due to the ligand binding can be examined using Kerr spectroscopy. Comparison between the signals of bound complex and that of uncorrelated protein/ligand demonstrated that binding action alone has

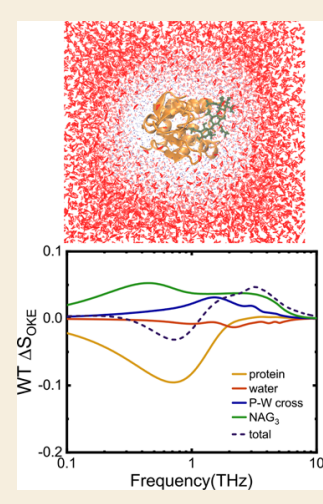
reflection in the OKE spectrum. Our study indicated OKE as a powerful terahertz probe for protein-ligand binding chemistry and dynamics.

KEYWORDS: ultrafast phenomenon, terahertz optical Kerr spectroscopy, protein ligand binding, nonlinear vibrational spectroscopy, confined water, terahertz collective vibration, Drude polarizable force field

\section{INTRODUCTION}

Protein-ligand interaction plays an important roles in life sciences, ${ }^{1-6}$ medicine, $^{7-11}$ as well as biotechnology. ${ }^{12-15}$ Besides the large-scale conformational dynamics, a binding reaction occurring at the active site of protein also couples with subtle protein structure fluctuations of potential biological significance. ${ }^{1,16-23}$ Certain collective motions in the enzymes, for instance, have time scales of hundred femtoseconds to couple picoseconds. These motions have been suggested to be able to guide the system along a specific path on a highly sophisticated potential energy surface and consequently facilitate chemical reactions and biological processes. ${ }^{24-26}$ Only sparse experimental investigations have been reported, however, on such motions due to the lack of probing tools. Their atomistic details and the knowledge of their involvement in biochemical activities are also largely absent.

Terahertz optical Kerr effect (THz OKE) spectroscopy allows the measurement of a terahertz depolarized Raman spectrum in the time domain and provides a powerful means for directly monitoring the concerted conformational fluctuations on a picosecond time scale. OKE spectroscopy has been widely used for the room temperature as well as supercool water, ${ }^{27-31}$ molecular liquids, ${ }^{32-36}$ ionic liquids ${ }^{37-39}$ and ionic solutions. ${ }^{40-44}$ Compared with other commonly used terahertz techniques, OKE suffers from neither liquid water absorption as in far-infrared and $\mathrm{THz}$ time-domain spectroscopy nor the Rayleigh peak as in spontaneous Raman scattering.

Recent advances in OKE spectroscopy allow further exploration of its applications to the solvated biomacromolecules. $^{20,45-48}$ Earlier studies on the protein films with different water contents suggested that OKE can detect the interplay of bound and free water in protein solvation dynamics. ${ }^{45} \mathrm{OKE}$ is also sensitive to the differences of protein ${ }^{47}$ and $\mathrm{DNA}^{48}$ structural motifs in solution. A recent OKE experiment ${ }^{20}$ further measured the difference signals between the solvated lysozyme and the solvated lysozyme-ligand complex.

Influence of ligand binding, and more generally of biochemical reactions and activities on the $\mathrm{THz}$ OKE spectra and the related picosecond dynamics, however, remains largely elusive due to the challenge of quantitatively interpreting the spectra with atomistic details. Molecular dynamic simulationbased spectrum modeling has great potential in elucidating the connections between the spectroscopic measurements and the related microscopic structural dynamics. Simulating the OKE

Received: August 15, 2021

Published: September 15, 2021 
spectra for an entire solvated protein complex using a consistent molecular model for both the molecular dynamic simulation force field and the spectrum (polarizability) calculation, however, was a difficult task not yet accomplished. $^{20}$

Here, we carried out a quantitative theoretical analysis to reveal the biochemical relevance of OKE spectroscopy to the protein-ligand binding activity. To that end, we calculated the OKE spectra of the solvated protein-ligand complexes, for the first time, using a consistent Drude polarizable model ${ }^{49}$ both as the underlying force field model for molecular simulation and as the polarizability model for spectrum calculation. An analytical multipole and induced dipole (MPID) scheme was adopted for the more efficient and accurate evaluation of the off-diagonal polarizability. MPID generates equivalent results with respect to the commonly used numerical Drude model calculations but avoids the expensive converge iterative treatment by using a direct matrix inversion, which therefore allows the Kerr spectrum modeling of large systems such as a solvated protein complex. A solvated hen egg-white lysozyme was used as the prototype of small enzymes, which is bound by a highly affinitive triacetylchitotriose $\left(\mathrm{NAG}_{3}\right)$ ligand (Figure 1, lower left). Excellent reproduction of the experimental spectra indicates the accuracy of our treatment.

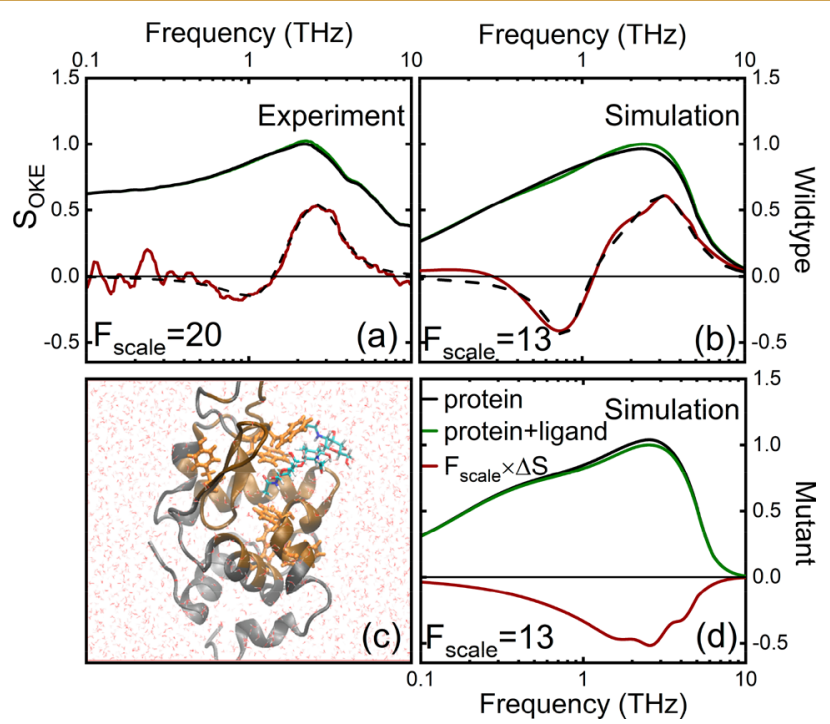

Figure 1. Experimental (a) and calculated (b) OKE signals of the solvated wildtype lysozyme with (green)/without (black) $\mathrm{NAG}_{3}$ binding, as well as their difference (red, enhanced by a factor, of 20 in the experimental and of 13 in the simulated result, for a better presentation). The black dash lines are the fitted signals using a Brownian Oscillator model (eq 1). (d) Calculated OKE signals for the mutated lysozyme D52S-Lz using the same color scheme as in (a) and (b). The difference signal (red) is enhanced by a factor of 13. (c) Cartoon representation of the solvated lysozyme-NAG . All the total signal of lysozyme solution peak are normalized to 1.0.

Decomposition of difference spectra between the signals of the samples with/without the ligand, for the wild type lysozyme as well as a mutated lysozyme D52S-Lz, ${ }^{50}$ identified the contributions of protein, water, and ligand to the overall difference spectra. Further analysis indicates that the subtle fluctuation of the aromatic residues dominates the protein component in the difference spectra, and the water molecules confined between the ligand and the protein cavity wall leads to the water component. A comparison between the signals of bound lysozyme-NAG 3 complex and a hypothetical system with uncorrelated protein and ligand further demonstrated that the action of binding alone indeed has reflection in the OKE spectrum.

\section{RESULTS AND DISCUSSION}

\subsection{Simulation of the OKE Signals for the Solvated Lysozyme with/without $\mathrm{NAG}_{3}$ Binding}

The experimental spectra ${ }^{20}$ for the solvated wild type lysozyme (PDB ID: $1 \mathrm{LZA}^{51}$ ) and lysozyme- $\mathrm{NAG}_{3}$ complex (PDB ID: $\left.1^{2 Z B}{ }^{51}\right)$ at $298 \mathrm{~K}\left(S_{\text {solv-prot }}^{\mathrm{WT}}\right.$ and $S_{\text {solv-prot-lig }}^{\mathrm{WT}}$ in the upper left panel of Figure 1) possess a broad asymmetric feature, with the maximum at $2.80 \mathrm{THz}$, a weak shoulder at $1.15 \mathrm{THz}$ and a longer tail extending to lower frequency. Binding of $\mathrm{NAG}_{3}$ generates an undulating feature in the difference spectrum $\Delta S^{\mathrm{WT}}=S_{\text {solv-prot }}^{\mathrm{WT}}-S_{\text {solv-prot-lig }}^{\mathrm{WT}}$ with a negative peak at $\sim 1.0$ $\mathrm{THz}$ and a positive peak $\sim 3.0 \mathrm{THz}$. The negative peak is weaker comparing to the positive peak.

Previous OKE calculations for the liquids and solutions of small molecules ${ }^{52,53}$ and ions ${ }^{54,55}$ as well as the solvated proteins ${ }^{56}$ were usually carried out based on the configuration ensemble generated using molecular dynamic simulation with nonpolarizable force fields. A separate polarizability model was then used to compute the total polarizability, in which the induced polarizability effect was often evaluated via dipoleinduced-dipole (DID) $)^{57}$ or point-polarizable-dipole (PPD) $)^{58}$ schemes. The spectra were consequently calculated as the time derivative of polarizability time correlation function. ${ }^{59}$ In these treatments, however, the effect of the possible inconsistency between the molecular dynamic simulation force field and the polarizability model is difficult to evaluate.

OKE simulations using polarizable force field consistently for both molecular dynamic simulation and the spectrum calculation were reported only recently ${ }^{60,61}$ for pure or mixed liquids, and these simulations were limited to the systems of small molecules. On the other hand, force field development efforts in the past decade yielded transferable and highly accurate polarizable models for biomacromolecules. ${ }^{62,63}$ This encouraged us to calculate the solvated lysozyme- $\mathrm{NAG}_{3} \mathrm{OKE}$ spectra using one consistent polarizable model.

Details of our simulations are provided in the Methods. Briefly, molecular dynamic simulations with the Drude polarizable model were carried out to generate the configuration ensembles of both solvated protein and protein-ligand complex systems. In the Drude polarizable model, induced dipole moment $\mu$ is indirectly provided via the relative position of the Drude particle. This allows efficient propagation of dynamics but renders the numerical calculation of polarizability tensor from definition $\alpha=\mu / E$ inefficient and a lack of accuracy. In particular, to evaluate the off-diagonal polarizability one has to compute the dipole response with an explicitly applied electric field $E$ in perpendicular directions, which could take up to 1000 self-consistent iterations to relax the positions of Drude particles. Recently, we established the equivalency between the Drude polarizable model and a multipole and induced dipole model, $^{64}$ which allows to evaluate the polarizability tensor analytically using direct matrix inversion with atomic polarizability and the Thole screening scheme ${ }^{57}$ adopted from the Drude FF model. Thole damping function in MPID was implemented within the quasi internal coordinates for spherical harmonic multipoles and 
truncated according to third order perturbation theory with optimized empirical coefficients. $^{65}$

The time correlation functions (TCFs) of the total polarizability were then calculated, and their time derivatives were Fourier transformed to generate the OKE signals in a frequency window between 0.1-10 THz. Our simulations (Figure 1b) nicely reproduced the main features of the experimental observations (Figure 1a), including the negative lobe at $\sim 1.0 \mathrm{THz}$ in $\Delta S^{\mathrm{WT}}$, and a stronger (comparing to the negative lobe) positive lobe at $\sim 3.0 \mathrm{THz}$.

The simulated spectra $\Delta S^{\mathrm{WT}}$ can be fitted by a pair of Brownian oscillators (Figure 1, dash line):

$$
\Delta S_{\mathrm{BO}}(\omega)=C_{1} \frac{\gamma_{1} \omega}{\left(\omega_{1}^{2}-\omega^{2}\right)^{2}+4 \gamma_{1}^{2} \omega^{2}}+C_{2} \frac{\gamma_{2} \omega}{\left(\omega_{2}^{2}-\omega^{2}\right)^{2}+4 \gamma_{2}^{2} \omega^{2}}
$$

Our Brownian oscillators fitting results are $\omega_{1}=0.83 \mathrm{THz}$, $\gamma_{1}=0.30 \mathrm{THz}, \omega_{2}=3.59 \mathrm{THz}, \gamma_{2}=1.91 \mathrm{THz}$, while the experimental results are $\omega_{1}=1.29 \mathrm{THz}, \gamma_{1}=0.64 \mathrm{THz}, \omega_{2}=$ $2.89 \mathrm{THz}, \gamma_{2}=1.38 \mathrm{THz}$. More details of Brownian oscillators fitting are shown in Figure S5 (see Supporting Information). The fitting parameter $\gamma$ is less than the frequency $\omega$ for each mode, suggesting that the vibrational modes involved are underdamped, which is again consistent with the experiment. ${ }^{20}$

To estimate the sensitivity of the OKE features in $\Delta S$ to different ligand binding conditions, we further calculated the OKE signals for a mutated lysozyme D52S-Lz (PDB ID: $\left.1 \mathrm{LSZ}^{66}\right)$ (Figure 1 lower right), in which the catalytic residue aspartic acid at position 52 was substituted with serine. This mutation induces only negligible change in the structure of protein cavity according to previous circular dichroism and fluorescence experiments ${ }^{50}$ and DFT calculations. ${ }^{67}$ On the other hand, instead of having an undulating shape as in the wildtype lysozyme, calculated $\Delta S^{\mathrm{MUT}}$ of the solvated D52S-Lz has a broad negative feature with the minimum at $\sim 3.0 \mathrm{THz}$.

$\mathrm{THz}$ OKE spectra studied herein originate from the cancellation of the contributions from protein, water, ligand, and their cross correlations. Previous molecular dynamics simulation studies on the solvated wildtype lysozyme- $\mathrm{NAG}_{3}$ complex $^{20}$ suggested that the vibrational motions within the 1.0-3.0 THz range involve the delocalized backbone torsional motions and the libration of the side chains. To further disentangle this cancellation and to reveal the molecular origin of the subtle $\mathrm{THz}$ OKE features in detail, and their relevance to the ligand binding, we next decomposed the difference signal $\Delta S$ into the contributions from different components.

\subsection{Decomposing the Difference OKE Spectra}

By writing the total polarizability as the sum of the contributions from different parts of the solvated biomolecular complex: $\Pi_{\text {total }}=\Pi_{\text {water }}+\Pi_{\text {protein }}+\Pi_{\text {ligand }}$, the overall difference signal $\Delta S$ can be decomposed into the contributions from protein, water, and ligand as well as their cross correlations. For both the wildtype and mutant complexes, Figure 2 presents the contributions to the signals $\Delta S$ from protein, water, protein-water correlation, and ligand, respectively. The very small contributions from the ligand-water and ligandprotein cross correlations (Figure S1) are not presented here. For the wildtype, the positive peak at $\sim 3.0 \mathrm{THz}$ in $\Delta S^{\mathrm{WT}}$ (purple dash) is mainly provided by the ligand component (green), while the negative peak at $\sim 1.0 \mathrm{THz}$ results from the cancellation of the negative protein component (yellow) and

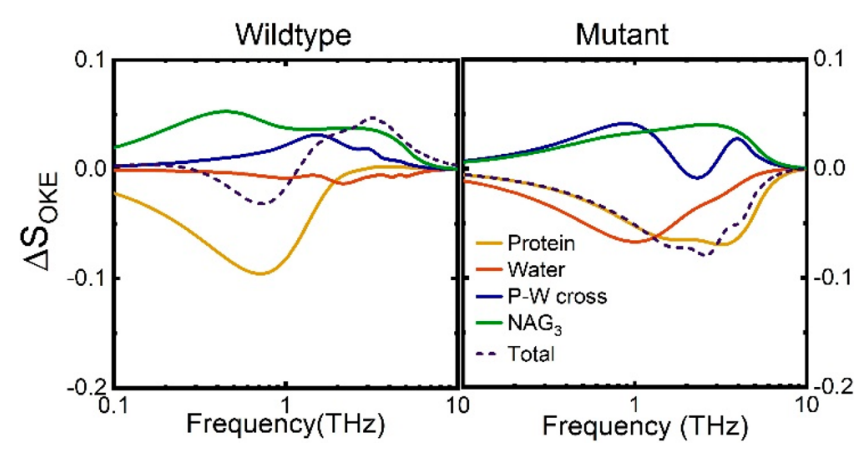

Figure 2. Contributions to $\Delta S$ (purple dash) of wildtype (left) and mutated (right) lysozyme from protein (yellow), water (red), protein-water cross-term (blue), and ligand (green) components, respectively.

the positive ligand (green) component and protein-water cross term (blue).

Protein contribution in the wildtype signal $\Delta S^{\mathrm{WT}}$ has a significant negative peak at $\sim 1.0 \mathrm{THz}$, which shifts to higher frequency in the mutant signal. Water contribution in the wildtype signal is negligible, while that in the mutant has a strong negative contribution. Protein as well as water contributions to $\Delta S$ come from the differences between the protein/protein-ligand spectra, while the ligand contribution comes only from the protein-ligand spectra. Therefore, although the ligand contribution is much smaller than those of water and protein in the protein-ligand spectra, their absolute magnitudes in $\Delta S$ become comparable.

In the wildtype signal (Figure S1 left), protein component has the most important contribution because this component has the largest absolute amplitude and the absence of it will lead to the most significant change of the spectral line shape. In this sense, the ligand component and the protein-water cross correlation term also have the fairly significant contributions, while the contributions from water as well as the proteinligand and water-ligand cross correlations are negligible in the wildtype signal. In the mutant signal (Figure S1 right) the observations are similar, but the water contribution becomes important (comparable to the protein component, more significant than the ligand component) as well. Since we want to understand how the changes in protein and water structural dynamics due to the ligand binding are reflected in the OKE signals, in the following we focus on analyzing the protein and water signals in $\Delta S$, revealing their molecular origins and establishing their connections to the ligand-binding action. Our analysis on the ligand component did not reveal a clear connection between the feature of this component and the ligand molecular detail. We therefore chose not to unfold the related discussion herein.

\subsection{Contributions of Protein in the Difference OKE Spectra}

For the wildtype, the protein component in the difference signal, $\Delta S_{\text {prot }}^{\mathrm{WT}}$ has a negative feature instead of an undulation (Figure 2, yellow). There is therefore no significant frequency shift between the protein component in the wildtype OKE signals with/without ligand. The calculated protein vibrational density of states (VDOS, Figure 3a,b) also appears to be invariant with/without the presence of ligand (similar observations are also reported in ref 20). 

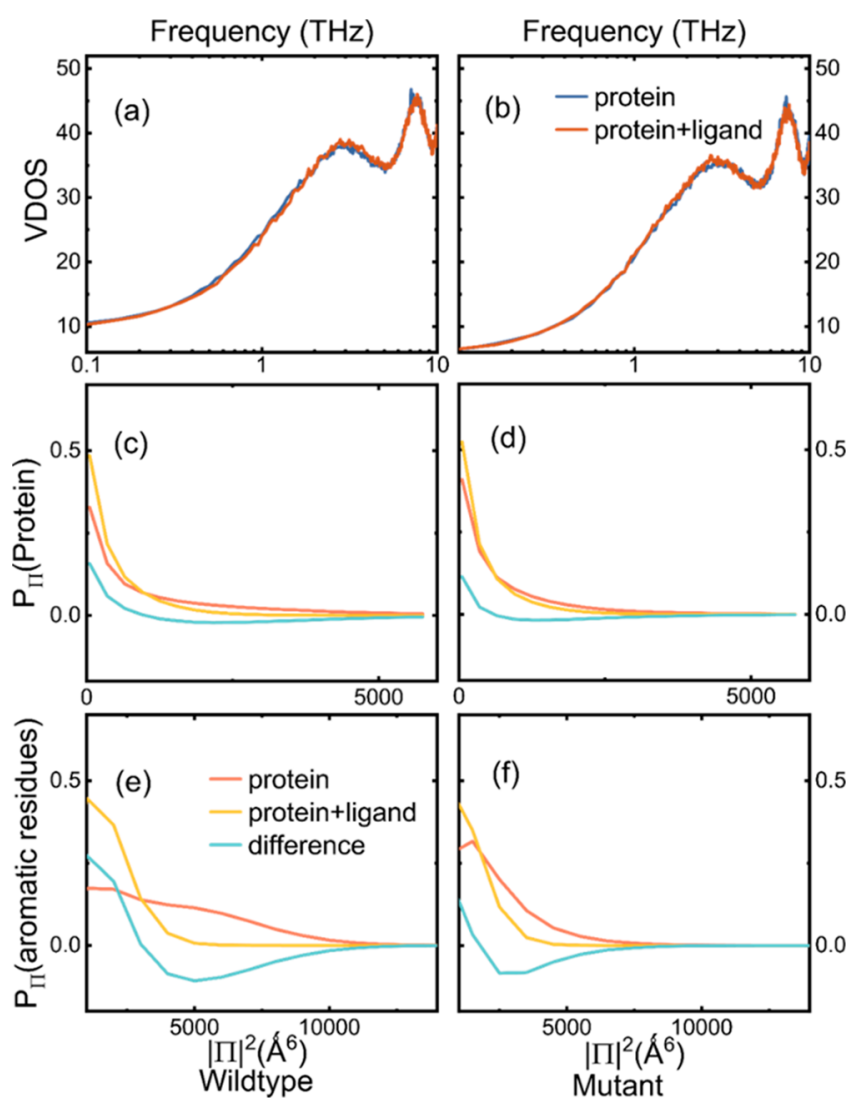

Figure 3. (a,b) Protein vibrational density of states for lysozyme without (blue) and with (red) ligand binding. (c,d) Distributions of $\left(\Pi_{\text {protein }}^{x y}\right)^{2}+\left(\Pi_{\text {protein }}^{y z}\right)^{2}+\left(\Pi_{\text {protein }}^{z x}\right)^{2}$ for the anisotropic components of polarizability of the whole protein. Orange (red) line is for the system with (without) ligand. Cyan line is the difference; (e,f) Similar distributions $\left(\Pi_{\text {aromatic }}^{x y}\right)^{2}+\left(\Pi_{\text {aromatic }}^{y z}\right)^{2}+\left(\Pi_{\text {aromatic }}^{z x}\right)^{2}$ for the aromatic residues only. The left panels are for the wildtype while the right panels are for the mutant.

OKE responses were calculated by averaging over the independent contributions from the correlation functions of off-diagonal polarizability elements $(x y, y z$, and $x z)$ :

$$
R(t) \propto \frac{\partial}{\partial t}\left\langle\Pi^{x y}(0) \Pi^{x y}(t)+\Pi^{y z}(0) \Pi^{y z}(t)+\Pi^{z x}(0) \Pi^{z x}(t)\right\rangle
$$

We can therefore estimate how the change of polarizability amplitude affect the signals by calculating the distributions of $\left(\Pi_{\text {protein }}^{x y}\right)^{2}+\left(\Pi_{\text {protein }}^{y z}\right)^{2}+\left(\Pi_{\text {protein }}^{z x}\right)^{2}$, the summation of offdiagonal components of the total protein polarizability (Figure $3 \mathrm{c}, \mathrm{d})$. This distribution shifts to the lower values in the protein-ligand complex with respect to that in the proteinonly system, indicating that the negative $\Delta S_{\text {prot }}$ in both wild type and mutant originate from the reduced total protein polarizability magnitudes.

To achieve a further microscopic insight, we divided the protein signal $\Delta S_{\text {prot }}$ into the contributions from the aromatic residues ( 3 phenylalanine, 3 tyrosine, 6 tryptophan, and 1 histidine) $\Delta S_{\text {prot-aromatic }}$ and those from the nonaromatic residues $\Delta S_{\text {prot-nonaromatic }}$. Figure 4 shows that the aromatic contribution (yellow dash) shapes the main feature of the protein signal (green), while the nonaromatic contribution (red dash) is much less significant. This is because the reorientational motions of the aromatic residues, which have the high polarizability anisotropy, generate the largest contributions to the OKE spectrum. We can also divide the

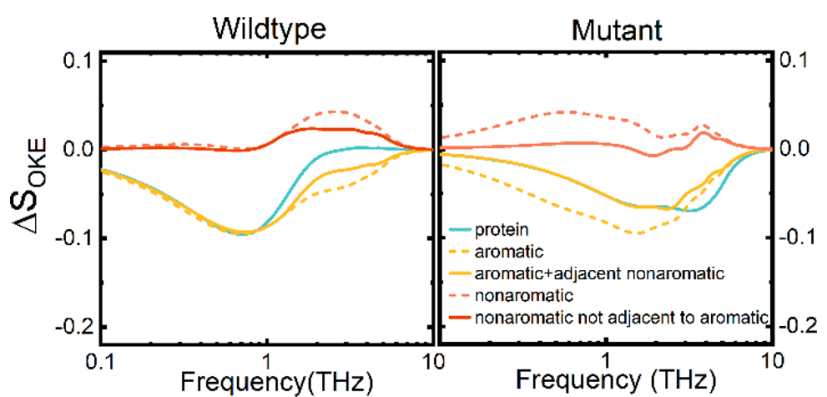

Figure 4. Total protein contributions $\Delta S_{\text {prot }}$ (green) in the difference OKE spectra of wildtype (left) and mutant (right) as well as the contributions from aromatic protein residues (yellow dash), nonaromatic protein residues (red dash), aromatic residue plus the adjacent nonaromatic residues (yellow solid), and the nonaromatic residues not adjacent to the aromatic residues (red solid).

protein signal into the contributions from (a) the aromatic residues plus the adjacent nonaromatic residues (yellow solid) and (b) other nonaromatic residues (red solid). The contribution from the residues in group (a) dominates even more in the overall protein signals. The difference protein signal $\Delta S_{\text {prot }}$ therefore mainly originates from the contributions of aromatic groups. Figure $3 \mathrm{e}, \mathrm{f}$ show the distribution of $\left(\Pi_{\text {aromatic }}^{x y}\right)^{2}+\left(\Pi_{\text {aromatic }}^{y z}\right)^{2}+\left(\Pi_{\text {aromatic }}^{z x}\right)^{2}$ for the off-diagonal components of the total polarizability of the aromatic protein residues only. The distribution for the ligand bound complex shifts to the lower values with respect to the corresponding part for the solvated protein only, suggesting a significant reduction of overall polarizability of the aromatic residues with the binding of ligand.

This polarizability reduction is the consequence of ligand binding on the relative spatial arrangements of these aromatic protein residues. Parts $a$ and $b$ of Figure 5 present the average dipole-dipole interaction amplitudes for each pair of aromatic groups in the solvated proteins (upper triangle) and the solvated protein + ligand complexes (lower triangle). For both the wildtype (Figure 5a) and the mutant (Figure 5b) samples, ligand binding leads to weaker interactions in a number of pairs (e.g., TRP108-TYR111, TRP28-TYR111, TRP62TRP63), suggesting a more "twisted" spatial arrangements of these aromatic groups. Furthermore, the fluctuations of these interactions (Figure 5c,d) are smaller in the ligand bound samples, which suggests that ligand binding makes the spatial arrangements of aromatic residues more rigid. The distributions of distance (upper panel in Figure S2) and the relative orientation (lower panel in Figure S2) between the dipoles of each aromatic group pair, on the other hand, show no significant shift to larger value with the ligand binding. The average value of distances changes from 14.55 to $14.66 \AA$ with ligand binding for the wildtype and from 14.65 to $14.62 \AA$ for the mutant. The average value of $\cos \theta$ between the dipole pairs changes from 0.027 to 0.036 with ligand binding for the wildtype and from 0.017 to 0.044 for the mutant. Therefore, instead of significantly changing the distributions of the relative distances and orientations between the aromatic residues, the ligand binding twists and stiffens their spatial arrangements in a more subtle way, which reduces the total polarizability of the protein and generates a broad negative peak in the protein signal $\Delta S_{\text {prot}}$.

The protein OKE signal (yellow line in Figure 2 right panel) in the mutant, $\Delta S_{\text {prot }}^{\mathrm{MUT}}$, is blue-shifted with respect to the 


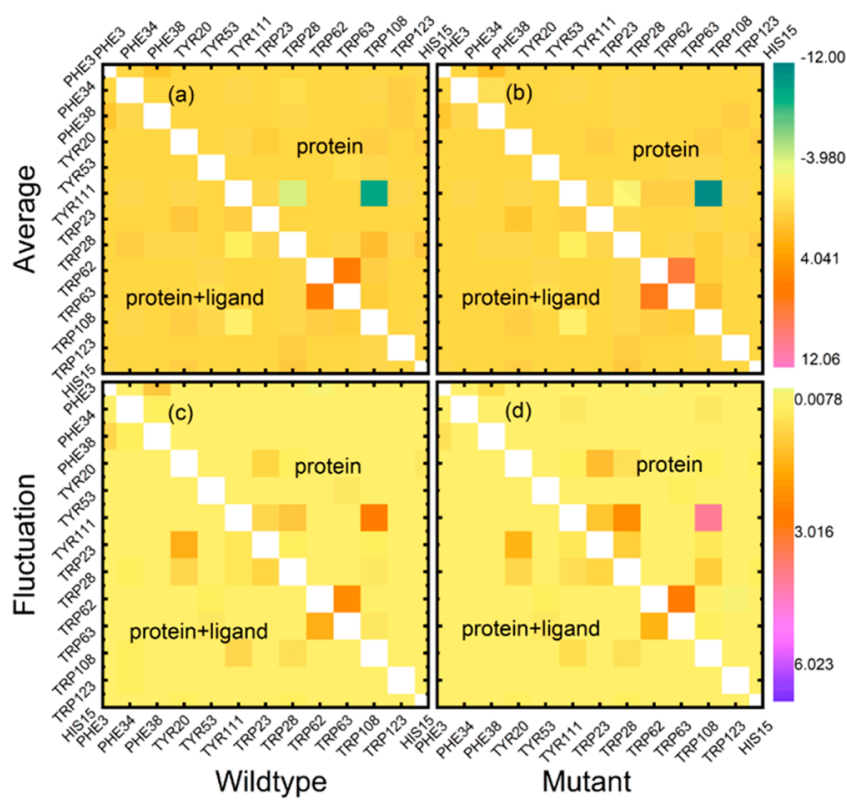

Figure 5. Dipole-dipole interactions between each pair of the 13 aromatic residues of the wildtype (left column) and mutant (right column). Upper row: the average values of the interactions. Lower row: the fluctuations of the interactions. Lower triangle regions in each panel are for the solvated protein + ligand while upper triangles are for the solvated protein only. The interaction difference of the two states is plotted in Figure S4 (see the Supporting Information).

wildtype signal $\Delta S_{\text {prot }}^{\mathrm{WT}}$ (yellow line in Figure 2 left panel). Comparisons between the lower triangles in Figure 5a,b and the lower triangles in Figure $5 \mathrm{c}$,d indicate that in the solvated protein + ligand complexes the average couplings as well as their fluctuations have similar patterns between the wildtype and the mutant. Significant differences of patterns, on the other hand, appear for the solvated protein-only systems (upper triangles in Figure 5a,b and the upper triangles in Figure 5c,d). The difference between the protein signals for the wildtype and mutant is therefore largely induced by the different spatial arrangement patterns and their fluctuations of the aromatic residues in the protein-only samples, which is indistinguishable in the circular dichroism and fluorescence experiments. ${ }^{50}$ These results revealed the potential of OKE as a useful $\mathrm{THz}$ vibrational probe for the subtle protein conformational fluctuation and dynamics.

\subsection{Contribution of Water in the Difference OKE Spectra}

Figure 2 shows that in the wildtype signal $\Delta S^{\mathrm{WT}}$ the contribution from water is negligible. In the mutant signal $\Delta S^{\mathrm{MUT}}$, on the other hand, the water component has a significant, broad negative feature. Further, if we compute the $\Delta S_{\text {water-outside-pocket }}^{\mathrm{MUT}}$ for the mutant sample, in which in every snapshot of trajectory only the water molecules outside the binding pocket are considered in the polarizability calculations, the signal again becomes negligible (Figure 6). The significant water signal of mutant therefore originates from ligand binding effect on the pocket water molecules.

The difference in the negligible wildtype $\Delta S_{\text {water }}^{\mathrm{WT}}$ and the stronger mutant $\Delta S_{\text {water }}^{\mathrm{MUT}}$ can therefore be explained in a qualitative manner: For the wildtype lysozyme, the bound ligand squeezes some hydration water originally inside the cavity out to the bulk. At the same time, the part of the ligand outside of the pocket is exposed to water and creates a new population of hydration water. These two effects balance with

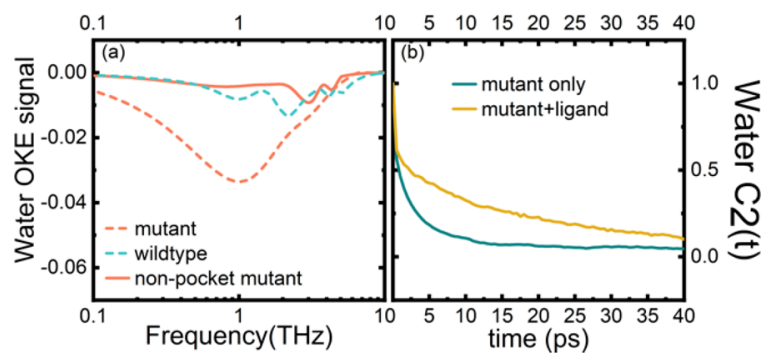

Figure 6. (a) Difference OKE signals of water in wildtype $\Delta S_{\text {water }}^{\mathrm{WT}}$ (cyan dash) and mutant $\Delta S_{\text {water }}^{\mathrm{MUT}}$ (red dash) systems, as well as the signal $\Delta S_{\text {water-outside-pocket }}^{\mathrm{MUT}}$ (red solid) calculated for mutant with only the water molecules outside of the binding pocket considered. (b) Rotational correlation functions of the water molecules adjacent to the protein residues 98-104 in the solvated mutant-only (blue) and mutant + ligand (yellow) systems.

each other and lead to an almost negligible $\Delta S_{\text {water }}^{\mathrm{WT}}$ For the mutant + ligand system, on the other hand, certain amount of water molecules are trapped near protein residues $98-104$ by the ligand residue NAG-2. These confined water molecules rotate much slower than the water molecules in the same region without ligand as well as the hydration water molecules near the exposed region of ligand, which leads to a significant negative feature in the frequency window inspected.

Figure 7 demonstrates the spatial distribution of water density $>1.0$ within the binding pocket for both wildtype and

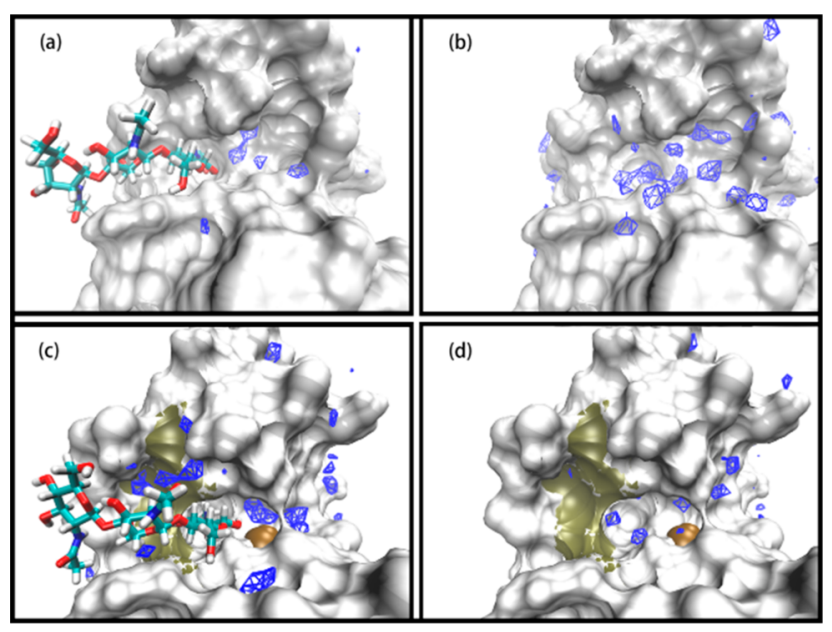

Figure 7. Water profile with density $>1.0$ (blue wireframes) inside the binding pocket for wildtype $(\mathrm{a}, \mathrm{b})$ and mutant $(\mathrm{c}, \mathrm{d})$. The ligand binding pocket in the presence $(a, c)$ and absence $(b, d)$ of the NAG ligand, respectively. The mutated residue is shown as a brown area in $(c, d)$. Tan area in $(c, d)$ is the region of mutant in which high density of water molecules are trapped between ligand and protein cavity. No such region is observed in the wildtype.

mutant with/without the ligand. In the solvated mutant + ligand complex (Figure 7c), a number of water molecules are trapped between the ligand residue NAG-2 and the cavity wall of protein residues 98-104 ( $\tan$ area), leading to a high water density in this region. For the mutant protein-only system (Figure 7d), water molecules in this region have much lower density, suggesting a much higher liquidity of these water molecules. The confined water molecules in the solvated mutant + ligand rotate much more slowly than those in the same region of the solvated mutant-only system (Figure $6 \mathrm{~b}$ ), as 
well as the water molecules in the bulk water and the external hydration shell of the proteins (Figure S3). Furthermore, for the wildtype protein + ligand system (Figure 7a), water between the ligand and the protein has much lower density.

OKE has long been used to study the orientational dynamics of confined water in the nanoporous media. ${ }^{68-70} \mathrm{We}$ demonstrated that carefully designed OKE experiments have the potential to probe the effect of confined small water clusters induced by the protein-ligand binding. This is a subtle feature in the molecular binding mechanism. It would be of interest to perform complementary measurements on these confined water molecules using, for instance, the NMR relaxation or neutron scattering techniques, and establish more comprehensive pictures on this topic.

\subsection{Direct Reflection of Protein-Ligand Binding in the OKE Signal}

An essential question relating to the chemical relevance of OKE is whether the binding action alone has direct reflection in the OKE spectrum. The spectra in Figure 1 provide an informative and experimentally accessible estimation to this question by measuring the difference between the signals of a solvated lysozyme and a solvated lysozyme-ligand complex. In this section, we attempted to address this issue from a different angle by comparing the signal of a solvated bound lysozyme$\mathrm{NAG}_{3}$ complex to the signal of a solvated lysozyme/ $\mathrm{NAG}_{3}$ system with no protein-ligand correlation. To that end, we constructed a hypothetic system with no protein-ligand correlation: two simulation systems with (1) one lysozyme and 12197 water molecules and (2) one $\mathrm{NAG}_{3}$ ligand molecule and 12197 water molecules are constructed, and their OKE signals $\left(S_{\text {solv-prot }}\right.$ and $\left.S_{\text {solv-lig }}\right)$ calculated. $S_{\text {solv-prot }}$ is decomposed into the protein contribution $S_{\text {solv-prot, the water }}^{\mathrm{p}}$ contribution $S_{\text {solv-prot }}^{\mathrm{w}}$ and the protein-water cross term $S_{\text {solv-prot. }}^{\text {pw }} S_{\text {solv-lig }}$ is decomposed into the ligand contribution

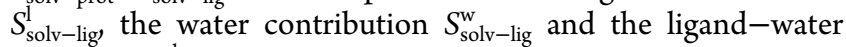

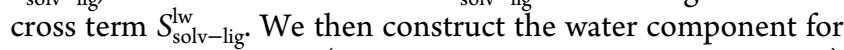
our hypothetic system (with uncorrelated protein and ligand) as $S_{\text {uncorr-prot-lig }}^{\mathrm{w}}=\left(S_{\text {solv-lig }}^{\mathrm{w}}+S_{\text {solv-prot }}^{\mathrm{w}}\right) / 2$ (since these two systems have same number of water molecules). The total signal of the hypothetic system is

$$
\begin{aligned}
S_{\text {uncorr-prot-lig }}= & S_{\text {uncorr-prot-lig }}^{w}+S_{\text {solv-prot }}^{p}+S_{\text {solv-prot }}^{p w} \\
& +S_{\text {solv-lig }}^{l}+S_{\text {solv-lig }}^{l w}
\end{aligned}
$$

$S_{\text {uncorr-prot-lig }}$ has the components of water, protein, ligand, and protein-water correlation as well as ligand-water correlation, but it does not have the protein-ligand correlation as in the signal of solvated protein-ligand system $S_{\text {solv-prot-lig. }}$ Furthermore, the protein and ligand contributions in $S_{\text {uncorr-prot-lig }}$ originate from the molecular structure and dynamics of isolated protein and ligand molecules, which are different from the bound complexes (reflected in $S_{\text {solv-prot-lig }}$ ). The difference $\Delta S_{\text {binding }}=S_{\text {uncorr-prot-lig }}-S_{\text {solv-prot-lig }}$ (yellow curve in Figure 8), therefore, to a certain extent, reflects the overall effect of binding action on the OKE signal. $\Delta S_{\text {binding }}$ has a significant feature peaked at $\sim 1 \mathrm{THz}$, which indicates that the protein-ligand binding action herein indeed has a nonnegligible reflection on the OKE spectrum. Note that $\Delta S_{\text {binding }}$ is different from the previously discussed undulating difference signal $\Delta S=S_{\text {solv-prot }}-S_{\text {solv-prot-lig }}$ (the red curve in Figure $1 \mathrm{~b}$ and the purple curve in Figure 8), since they are contributed by different spectral components. Both of these two spectral

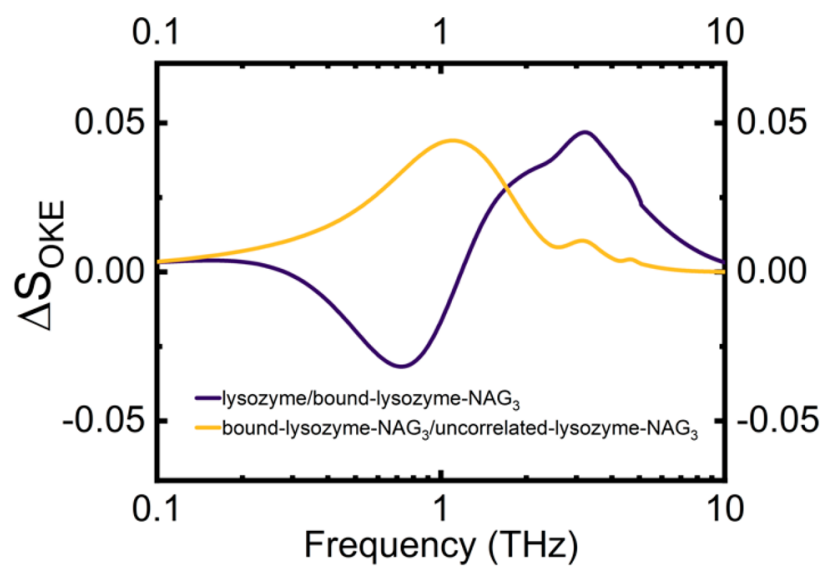

Figure 8. Difference OKE signals between the spectra of (purple) wildtype lysozyme with/without ligand binding and (yellow) wildtype lysozyme with ligand binding and the hypothetical system with no lysozyme-ligand correlation.

observables suggest the non-negligible biochemical relevance of Kerr spectrum to the protein ligand binding activity.

\section{CONCLUSIONS}

In this work we revealed, with great molecular details, an important biochemical relevance of OKE spectroscopy as a powerful $\mathrm{THz}$ vibrational probe for the protein-ligand binding chemistry and dynamics. Our discoveries are based on the first theoretical simulation of the solvated protein-ligand complex OKE spectra using a Drude polarizable model consistently for the molecular dynamic simulation force field as well as the polarizability calculations. Analysis on the simulated spectra illustrated that OKE signals contain rich molecular details about the binding effect on the subtle change of aromatic protein residues' spatial distributions and on the confined water molecules between ligand and protein cavity. A comparison between the signals of bound protein-ligand complex and a hypothetical system with uncorrelated protein and ligand further demonstrated that binding action alone has reflection in the OKE spectrum. Our study therefore demonstrated the great potential of OKE technique, in general, as a powerful characterization tool for the molecular dynamics related to the biochemical activities.

\section{METHODS}

\subsection{Molecular Dynamic Simulations}

The molecular dynamic simulations are carried out using Drude ${ }^{71}$ polarizable model with $\mathrm{NAMD}^{72}$ software. The crystal structures $\left(1 \mathrm{LZA} / 1 \mathrm{LZB}^{51}\right.$ ) of the hen egg-white lysozyme (HEWL) with/ without $\mathrm{NAG}_{3}$ are obtain from PDB databank.

Both of the nonbound and bound lysozyme complexes are solvated using a cubic water box of $72.56 \mathrm{~A}^{3}$ with 12197 SWM4-NDP water molecules. The Drude-2013 protein force field ${ }^{49}$ was used to model lysozyme. For the NAG ligand, although there is a recent work of Drude parametrization, ${ }^{73}$ there is no force field parameters when we start our simulations so a Drude model for NAG is developed by ourselves following the general parametrization procedure of Drude force fields ${ }^{63}$ (parameters provided in SI). Lysozyme contains 8 positive charges, and $8 \mathrm{Cl}^{-}$anions using the model described in ref 74 are therefore added to create a system neutral in charge. Our simulation system has a dilute concentration $(0.005 \mathrm{~mol} / \mathrm{L})$ very similar to that used in the simulation $(0.007 \mathrm{~mol} / \mathrm{L})$ and the experiment $(0.015 \mathrm{~mol} / \mathrm{L})$ of ref 20 . A previous experiment ${ }^{75}$ 
suggested that the solvated lysozyme $\mathrm{THz}$ OKE signals are very similar in the dilute solutions.

A 100-step energy minimization was carried out using the Adopted Basis Newton-Raphson (ABNR) ${ }^{76}$ for the Drude particles at first, and then the entire system was relaxed using Steepest Descent ${ }^{77}$ (SD) for 100 steps; at last, a 100 -step ABNR minimization was again used to reach the local minima.

A $10 \mathrm{~ns}$ NPT ensemble equilibration was then carried out to obtain the proper size of the simulation box. In the NPT simulation, the equation of motion was integrated with a time step of $1 \mathrm{fs}$ using an extended Lagrangian method with dual-Langevin thermostats, ${ }^{78}$ which maintained the system pressure at 1 atm with the constant temperature of $300 \mathrm{~K}$. A $10 \mathrm{~ns}$ NVE simulation using the temperature rescaling scheme was then carried out to stabilize the system temperature at $\sim 300 \mathrm{~K}$. A $300 \mathrm{~ns}$ NVE production run was then performed to generate the conformational trajectory for the later calculation of fluctuating polarizability.

van der Waals interactions were treated with the cutoff method $\left(r_{\mathrm{vdW}}=12 \AA\right)$, with L-J correction to maintain the system energy conservation. Nonbond exclusion were 1-4 scaling with factor of 1.0. Coulomb interactions were treated using the PME method $\left(r_{\mathrm{C}}=12\right.$ $\AA$ ) and the Verlet potential-shift approach $\left(r_{\text {switch }}=10 \AA\right)$. The neighbor list was updated every two steps within a pair list distance of $16 \AA$, in which electrostatics and van der Waals interactions for all the pairs were calculated. Water molecules were kept rigid during the simulations using the SETTLE algorithm. The Drude hardwall ${ }^{79}$ was used with a distance of $0.25 \AA$ and the Drude damping parameter for the Langevin integrator, used in NAMD, is $20.0 \mathrm{ps}^{-1}$. An additional quartic restraining potential is applied to a Drude oscillator if its length exceeds $0.25 \AA$.

\subsection{Calculations of OKE Spectra}

The overall optical response $R_{\text {tot }}(t)$ of the OKE signal can be decomposed into the contributions from the electrons $R_{\text {ele }}(t)$ and the nuclei $R(t) .{ }^{80}$ The instantaneous contribution from electrons $R_{\text {ele }}(t)$ is usually subtracted in the presented experimental data. Herein, as in most of the theoretical works on OKE spectrum simulation, only $R(t)$ containing inter- and intramolecular dynamical information in the low-frequency range is considered. For isotropic medium, $R(t)$ can be calculated as the time derivative of the two-point time correlation function of the anisotropic part of the many-body polarizability tensor $\Pi(t)^{81-83}$

$$
R(t)=-\frac{1}{k T} \frac{\partial}{\partial t} \Psi^{\text {aniso }}(t)
$$

with

$$
\Psi^{\text {aniso }}(t)=\frac{\left\langle\Pi^{x y}(0) \Pi^{x y}(t)\right\rangle+\left\langle\Pi^{y z}(0) \Pi^{y z}(t)\right\rangle+\left\langle\Pi^{x z}(0) \Pi^{x z}(t)\right\rangle}{\frac{1}{15} N \gamma^{2}}
$$

Here, $N$ is the number density of molecules and $\gamma$ is the ideal polarizability anisotropy of the isolated molecule. $\Pi^{i j}(t)$ is the sum of total off-diagonal polarizability along the $i-j$ directions at time $t(i \neq$ $j)$, generated using the molecular dynamics simulation trajectories. ${ }^{54}$ The same Drude polarizable model used in the molecular dynamic simulation for protein, ${ }^{49}$ water(SWM4-NDP), ${ }^{84}$ as well as ligand parameter/structure information was used herein for the polarizability calculations. $(t):$

The MPID scheme was used to calculate the total polarizability $\Pi$

$$
\Pi=\Pi^{\text {iso }}+\Pi^{\text {aniso }}=C\left(\sum_{i, j} \frac{d_{1}}{r_{i j}^{3}}+\mathbf{U}^{\dagger}(r) \frac{2 d_{0}+d_{1}}{r_{i j}^{3}} \mathbf{U}(r)\right)
$$

Here, $C$ is the unit conversion constant factor, $r_{i j}$ is the distance between the two polarizable atoms $i$ and $j$, and $d_{0}$ and $d_{1}$ are the numerators of Thole modified dipole tensor as defined in eqs S2 and S3. $\Pi^{\text {iso }}$ and $\Pi^{\text {aniso }}$ are the diagonal polarizability and off-diagonal polarizability, respectively. The quasi internal (QI) coordinates transformation $\mathbf{U}(r)$ is applied to rotate the molecular anisotropic polarizability into the laboratory frame. The Thole model of modified dipole interaction ${ }^{57}$ was used to calculate molecular polarizabilities from isotropic atomic polarizabilities to solve the polarization catastrophe (see the Supporting Information for details).

Time correlation functions of polarizability were fitted using function $R(t)$ :

$$
R(t)=A_{1} \exp \left(-\frac{t}{\tau_{1}}\right)+A_{2} \exp \left(-\frac{t}{\tau_{2}}\right)+C_{3} \exp \left[-\left(\frac{t}{\tau_{3}}\right)^{\beta_{3}}\right]
$$

and the fitted parameters are given in the Supporting Information. Here, $\tau_{1}>\tau_{2}>\tau_{3}$, the two slow terms $A_{1} \exp \left(-\frac{t}{\tau_{1}}\right)$ and $A_{2} \exp \left(-\frac{t}{\tau_{2}}\right)$ ( $\tau_{1}$ is about $6.0-9.0 \mathrm{~ns}, \tau_{2}$ is about $50.0-60.0 \mathrm{ps}$ ) were fitted using the single exponential (Debye) functions while the fast term within the $\mathrm{THz}$ range using a stretched exponential function (KohlrauschWilliams-Watts) $C_{3} \exp \left[-\left(\frac{t}{\tau_{3}}\right)^{\beta_{3}}\right]$. The first two slow terms were then subtracted from the correlation function since they are related to the processes outside the time window of interest. A damping procedure using the Standard Blackman window function ${ }^{85}$ was then applied to smoothen the remaining components of the polarizability correlation function. First, the derivative of the damped correlation function was calculated using finite-difference. A 1000-point sequence discrete Fourier transforms was then used to generate the frequency domain OKE signal via the FFTW3 package. ${ }^{86}$

$$
S(\omega)=\int_{0}^{\infty} \mathrm{d} t \mathrm{e}^{-i \omega t} R^{\prime}(t)=\int_{0}^{\infty} \mathrm{d} t \mathrm{e}^{-i \omega t} \frac{\partial R(t)}{\partial t}
$$

\section{ASSOCIATED CONTENT}

\section{Supporting Information}

The Supporting Information is available free of charge at https://pubs.acs.org/doi/10.1021/jacsau.1c00356.

Discussion of Thole damping model in polarizability calculation; discussion of fitting parameters for polarizability time correlation function; discussion of protocol used for the vibrational density of states calculations; full decomposition of simulated Kerr signals for both wildtype and mutant; distributions of distance and orientation between the dipoles of the aromatic residue pairs; rotational correlation functions of trapped as well as bulk and shell water in the solvated mutant + ligand system; dipole-dipole interactions difference between the aromatic residues; Brownian oscillator fitting data for the wildtype signals (PDF)

\section{AUTHOR INFORMATION}

\section{Corresponding Authors}

Wei Zhuang - State Key Laboratory of Structural Chemistry, Fujian Institute of Research on the Structure of Matter, Chinese Academy of Sciences, Fuzhou, Fujian 35000, China; Institute of Urban Environment, Chinese Academy of Sciences, XiaMen, Fujian 361021, China; 이이.org/ 0000-0003-4106-0985; Email: wzhuang@fjirsm.ac.cn

Jing Huang - Westlake Laboratory of Life Sciences and Biomedicine, Key Laboratory of Structural Biology of Zhejiang Province, School of Life Sciences, Westlake University, Hangzhou, Zhejiang 310024, China; 다. orcid.org/0000-0001-9639-2907; Email: huangjing@ westlake.edu.cn 


\section{Author}

Zhijun Pan - State Key Laboratory of Structural Chemistry, Fujian Institute of Research on the Structure of Matter, Chinese Academy of Sciences, Fuzhou, Fujian 35000, China; Shenzhen Bay Laboratory, Guangming District, Shenzhen 518107, China

Complete contact information is available at: https://pubs.acs.org/10.1021/jacsau.1c00356

\section{Notes}

The authors declare no competing financial interest.

\section{ACKNOWLEDGMENTS}

This material is based upon work supported by the NSFC Grants (21873101, 21803057), the FJIRSM\&IUE Joint Research Fund (No. RHZX-2019-002) and the Zhejiang NSF Grant LR19B030001. Simulations were carried out at Westlake University Supercomputer Center and we thank related technical assistance.

\section{REFERENCES}

(1) Zidek, L.; Novotny, M. V.; Stone, M. J. Increased protein backbone conformational entropy upon hydrophobic ligand binding. Nat. Struct. Biol. 1999, 6 (12), 1118-1121.

(2) Benkovic, S. J.; Hammes-Schiffer, S. A perspective on enzyme catalysis. Science 2003, 301 (5637), 1196-1202.

(3) Ye, S. X.; Zaitseva, E.; Caltabiano, G.; Schertler, G. F. X.; Sakmar, T. P.; Deupi, X.; Vogel, R. Tracking G-protein-coupled receptor activation using genetically encoded infrared probes. Nature 2010, 464 (7293), 1386-U14.

(4) Ryde, U.; Soderhjelm, P. Ligand-Binding Affinity Estimates Supported by Quantum-Mechanical Methods. Chem. Rev. 2016, 116 (9), 5520-5566.

(5) Wang, W.; Donini, O.; Reyes, C. M.; Kollman, P. A. Biomolecular simulations: Recent developments in force fields, simulations of enzyme catalysis, protein-ligand, protein-protein, and protein-nucleic acid noncovalent interactions. Annu. Rev. Biophys. Biomol. Struct. 2001, 30, 211-243.

(6) Baglia, R. A.; Zaragoza, J. P. T.; Goldberg, D. P. Biomimetic Reactivity of Oxygen-Derived Manganese and Iron Porphyrinoid Complexes. Chem. Rev. 2017, 117 (21), 13320-13352.

(7) Wang, E.; Sun, H.; Wang, J.; Wang, Z.; Liu, H.; Zhang, J. Z. H.; Hou, T. End-Point Binding Free Energy Calculation with MM/PBSA and MM/GBSA: Strategies and Applications in Drug Design. Chem. Rev. 2019, 119 (16), 9478-9508.

(8) Hudson, K. L.; Bartlett, G. J.; Diehl, R. C.; Agirre, J.; Gallagher, T.; Kiessling, L. L.; Woolfson, D. N. Carbohydrate-Aromatic Interactions in Proteins. J. Am. Chem. Soc. 2015, 137 (48), 1515215160 .

(9) Diehl, C.; Engstrom, O.; Delaine, T.; Hakansson, M.; Genheden, S.; Modig, K.; Leffler, H.; Ryde, U.; Nilsson, U. J.; Akke, M. Protein Flexibility and Conformational Entropy in Ligand Design Targeting the Carbohydrate Recognition Domain of Galectin-3. J. Am. Chem. Soc. 2010, 132 (41), 14577-14589.

(10) Rostock, L.; Driller, R.; Gratz, S.; Kerwat, D.; von Eckardstein, L.; Petras, D.; Kunert, M.; Alings, C.; Schmitt, F. J.; Friedrich, T.; Wahl, M. C.; Loll, B.; Mainz, A.; Sussmuth, R. D. Molecular insights into antibiotic resistance - how a binding protein traps albicidin. Nat. Commun. 2018, DOI: 10.1038/s41467-018-05551-4.

(11) Butcher, D. T.; Alliston, T.; Weaver, V. M. A tense situation: forcing tumour progression. Nat. Rev. Cancer 2009, 9 (2), 108-122.

(12) Bao, G.; Suresh, S. Cell and molecular mechanics of biological materials. Nat. Mater. 2003, 2 (11), 715-725.

(13) Herrmann, H.; Bar, H.; Kreplak, L.; Strelkov, S. V.; Aebi, U. Intermediate filaments: from cell architecture to nanomechanics. Nat. Rev. Mol. Cell Biol. 2007, 8 (7), 562-573.
(14) Li, P.; Mao, Z. M.; Peng, Z. L.; Zhou, L. L.; Chen, Y. C.; Huang, P. H.; Truica, C. I.; Drabick, J. J.; El-Deiry, W. S.; Dao, M.; Suresh, S.; Huang, T. J. Acoustic separation of circulating tumor cells. Proc. Natl. Acad. Sci. U. S. A. 2015, 112 (16), 4970-4975.

(15) Kim, D. H.; Wong, P. K.; Park, J.; Levchenko, A.; Sun, Y. Microengineered Platforms for Cell Mechanobiology. Annu. Rev. Biomed. Eng. 2009, 11, 203-233.

(16) Suydam, I. T.; Snow, C. D.; Pande, V. S.; Boxer, S. G. Electric fields at the active site of an enzyme: Direct comparison of experiment with theory. Science 2006, 313 (5784), 200-204.

(17) Eisenmesser, E. Z.; Millet, O.; Labeikovsky, W.; Korzhnev, D. M.; Wolf-Watz, M.; Bosco, D. A.; Skalicky, J. J.; Kay, L. E.; Kern, D. Intrinsic dynamics of an enzyme underlies catalysis. Nature 2005, 438 (7064), 117-121.

(18) Balog, E.; Becker, T.; Oettl, M.; Lechner, R.; Daniel, R.; Finney, J.; Smith, J. C. Direct determination of vibrational density of states change on ligand binding to a protein. Phys. Rev. Lett. 2004, 93 (2), 4.

(19) Moorman, V. R.; Valentine, K. G.; Wand, A. J. The dynamical response of hen egg white lysozyme to the binding of a carbohydrate ligand. Protein Sci. 2012, 21 (7), 1066-1073.

(20) Turton, D. A.; Senn, H. M.; Harwood, T.; Lapthorn, A. J.; Ellis, E. M.; Wynne, K. Terahertz underdamped vibrational motion governs protein-ligand binding in solution. Nat. Commun. 2014, 5, 3999.

(21) Woods, K. N. THz time scale structural rearrangements and binding modes in lysozyme-ligand interactions. J. Biol. Phys. 2014, 40 (2), 121-137.

(22) MacRaild, C. A.; Daranas, A. H.; Bronowska, A.; Homans, S. W. Global changes in local protein dynamics reduce the entropic cost of carbohydrate binding in the arabinose-binding protein. J. Mol. Biol. 2007, 368 (3), 822-832.

(23) Nesmelova, I. V.; Ermakova, E.; Daragan, V. A.; Pang, M.; Menendez, M.; Lagartera, L.; Solis, D.; Baum, L. G.; Mayo, K. H. Lactose Binding to Galectin-1 Modulates Structural Dynamics, Increases Conformational Entropy, and Occurs with Apparent Negative Cooperativity. J. Mol. Biol. 2010, 397 (5), 1209-1230.

(24) Deak, J.; Chin, H. L.; Lewis, C. M.; Miller, R. J. D. Ultrafast phase grating studies of heme proteins: Observation of the lowfrequency modes directing functionally important protein motions. J. Phys. Chem. B 1998, 102 (34), 6621-6634.

(25) Davydov, A. S. Theory of Contraction of Proteins Under Their Excitation. J. Theor. Biol. 1973, 38 (3), 559-569.

(26) Liu, D.; Chu, X. Q.; Lagi, M.; Zhang, Y.; Fratini, E.; Baglioni, P.; Alatas, A.; Said, A.; Alp, E.; Chen, S. H. Studies of phononlike lowenergy excitations of protein molecules by inelastic X-ray scattering. Phys. Rev. Lett. 2008, 101 (13), 4.

(27) Torre, R.; Bartolini, P.; Righini, R. Structural relaxation in supercooled water by time-resolved spectroscopy. Nature 2004, 428, 296-299.

(28) Turton, D. A.; Corsaro, C.; Martin, D. F.; Mallamace, F.; Wynne, K. The dynamic crossover in water does not require bulk water. Phys. Chem. Chem. Phys. 2012, 14, 8067-8073.

(29) Taschin, A.; Bartolini, P.; Eramo, R.; Righini, R.; Torre, R. Evidence of two distinct local structures of water from ambient to supercooled conditions. Nat. Commun. 2013, 4 (1), 2401.

(30) Bakker, H. J.; Skinner, J. L. Vibrational Spectroscopy as a Probe of Structure and Dynamics in Liquid Water. Chem. Rev. 2010, 110 (3), $1498-1517$.

(31) Winkler, K.; Lindner, J.; Bursing, H.; Vohringer, P. Ultrafast Raman-induced Kerr-effect of water: Single molecule versus collective motions. J. Chem. Phys. 2000, 113 (11), 4674-4682.

(32) Shirota, H.; Fujisawa, T.; Fukazawa, H.; Nishikawa, K. Ultrafast Dynamics in Aprotic Molecular Liquids: A Femtosecond RamanInduced Kerr Effect Spectroscopic Study. Bull. Chem. Soc. Jpn. 2009, 82 (11), 1347-1366.

(33) Kakinuma, S.; Shirota, H. Dynamic Kerr Effect Study on SixMembered-Ring Molecular Liquids: Benzene, 1,3-Cyclohexadiene, 1,4-Cyclohexadiene, Cyclohexene, and Cyclohexane. J. Phys. Chem. B 2015, 119 (13), 4713-4724. 
(34) Brodin, A.; Rossler, E. A. Depolarized light scattering versus optical Kerr effect. II. Insight into the dynamic susceptibility of molecular liquids. J. Chem. Phys. 2007, 126 (24), 244508.

(35) Hunt, N. T.; Jaye, A. A.; Meech, S. R. Ultrafast dynamics in complex fluids observed through the ultrafast optically-heterodynedetected optical-Kerr-effect (OHD-OKE). Phys. Chem. Chem. Phys. 2007, 9 (18), 2167-2180.

(36) Kinoshita, S.; Kai, Y.; Yamaguchi, M.; Yagi, T. Direct Comparison between Ultrafast Optical Kerr Effect and HighResolution Light Scattering Spectroscopy. Phys. Rev. Lett. 1995, 75 (1), 148-151.

(37) Shirota, H. Comparison of Low-Frequency Spectra between Aromatic and Nonaromatic Cation Based Ionic Liquids Using Femtosecond Raman-Induced Kerr Effect Spectroscopy. ChemPhysChem 2012, 13 (7), 1638-1648.

(38) Giraud, G.; Gordon, C. M.; Dunkin, I. R.; Wynne, K. The effects of anion and cation substitution on the ultrafast solvent dynamics of ionic liquids: A time-resolved optical Kerr-effect spectroscopic study. J. Chem. Phys. 2003, 119 (1), 464-477.

(39) Paluch, A. S.; Vitter, C. A.; Shah, J. K.; Maginn, E. J. A comparison of the solvation thermodynamics of amino acid analogues in water, 1-octanol and 1-n-alkyl-3-methylimidazolium bis(trifluoromethylsulfonyl)imide ionic liquids by molecular simulation. J. Chem. Phys. 2012, 137 (18), 184504.

(40) Palombo, F.; Heisler, I. A.; Hribar-Lee, B.; Meech, S. R. Tuning the Hydrophobic Interaction: Ultrafast Optical Kerr Effect Study of Aqueous Ionene Solutions. J. Phys. Chem. B 2015, 119 (29), 89008908.

(41) Foggi, P.; Bellini, M.; Kien, D. P.; Vercuque, I.; Righini, R. Relaxation Dynamics of Water and $\mathrm{HCl}$ Aqueous Solutions Measured by Time-Resolved Optical Kerr Effect. J. Phys. Chem. A 1997, 101, 7029-7035.

(42) Turton, D. A.; Hunger, J.; Hefter, G.; Buchner, R.; Wynne, K. Glasslike behavior in aqueous electrolyte solutions. J. Chem. Phys. 2008, 128, 161102 .

(43) Heisler, I. A.; Meech, S. R. Low-Frequency Modes of Aqueous Alkali Halide Solutions: Glimpsing the Hydrogen Bonding Vibration. Science 2010, 327, 857-860.

(44) Heisler, I. A.; Mazur, K.; Meech, S. R. Low-Frequency Modes of Aqueous Alkali Halide Solutions: An Ultrafast Optical Kerr Effect Study. J. Phys. Chem. B 2011, 115, 1863-1873.

(45) Eaves, J. D.; Fecko, C. J.; Stevens, A. L.; Peng, P.; Tokmakoff, A. Polarization-selective femtosecond Raman spectroscopy of lowfrequency motions in hydrated protein films. Chem. Phys. Lett. 2003, $376(1-2), 20-25$.

(46) Mazur, K.; Heisler, I. A.; Meech, S. R. Water Dynamics at Protein Interfaces: Ultrafast Optical Kerr Effect Study. J. Phys. Chem. A 2012, 116 (11), 2678-2685.

(47) Giraud, G.; Karolin, J.; Wynne, K. Low-frequency modes of peptides and globular proteins in solution observed by ultrafast OHDRIKES Spectroscopy. Biophys. J. 2003, 85 (3), 1903-1913.

(48) González-Jiménez, M.; Ramakrishnan, G.; Harwood, T.; Lapthorn, A. J.; Kelly, S. M.; Ellis, E. M.; Wynne, K. Observation of coherent delocalized phonon-like modes in DNA under physiological conditions. Nat. Commun. 2016, 7, 11799-11799.

(49) Lopes, P. E. M.; Huang, J.; Shim, J.; Luo, Y.; Li, H.; Roux, B.; MacKerell, A. D., Jr. Polarizable Force Field for Peptides and Proteins Based on the Classical Drude Oscillator. J. Chem. Theory Comput. 2013, 9 (12), 5430-5449.

(50) Ibrahim, H. R.; Matsuzaki, T.; Aoki, T. Genetic evidence that antibacterial activity of lysozyme is independent of its catalytic function. FEBS Lett. 2001, 506 (1), 27-32.

(51) Maenaka, K.; Matsushima, M.; Song, H.; Sunada, F.; Watanabe, K.; Kumagai, I. DISSECTION OF PROTEIN-CARBOHYDRATE INTERACTIONS IN MUTANT HEN EGG-WHITE LYSOZYME COMPLEXES AND THEIR HYDROLYTIC ACTIVITY. J. Mol. Biol. 1995, 247 (2), 281-293.

(52) Fecko, C. J.; Eaves, J. D.; Tokmakoff, A. Isotropic and anisotropic Raman scattering from molecular liquids measured by spatially masked optical Kerr effect spectroscopy. J. Chem. Phys. 2002, 117 (3), 1139-1154

(53) Elola, M. D.; Ladanyi, B. M. Intermolecular polarizability dynamics of aqueous formamide liquid mixtures studied by molecular dynamics simulations. J. Chem. Phys. 2007, 126 (8), 13.

(54) Zhang, R.; Zhuang, W. Cation Effect in the Ionic Solution Optical Kerr Effect Measurements: A Simulation Study. J. Chem. Phys. 2014, 140, 054507.

(55) Hou, M.; Lu, R.; Yu, A. Polarizability series of aqueous polyatomic anions revealed by femtosecond Kerr effect spectroscopy. RSC Adv. 2014, 4, 23078-23083.

(56) Martin, D. R.; Matyushov, D. V. Hydration shells of proteins probed by depolarized light scattering and dielectric spectroscopy: Orientational structure is significant, positional structure is not. $J$. Chem. Phys. 2014, 141 (22), 22D501.

(57) Thole, B. T. Molecular polarizabilities calculated with a modified dipole interaction. Chem. Phys. 1981, 59, 341-350.

(58) Sala, J.; Guàrdia, E.; Martí, J. Effects of concentration on structure, dielectric, and dynamic properties of aqueous $\mathrm{NaCl}$ solutions using a polarizable model. J. Chem. Phys. 2010, 132, 214505.

(59) Guillot, B. A Molecular-Dynamics Study of the Far InfraredSpectrum of Liquid Water. J. Chem. Phys. 1991, 95 (3), 1543-1551.

(60) Polok, K.; Idrissi, A.; Gadomski, W. Low frequency response of methanol/acetone mixtures: Optical Kerr effect and molecular dynamics simulations. J. Mol. Liq. 2012, 176, 29-32.

(61) Polok, K. Simulations of the OKE Response in Simple Liquids Using a Polarizable and a Nonpolarizable Force Field. J. Phys. Chem. B 2018, 122 (5), 1638-1654.

(62) Albaugh, A.; Boateng, H. A.; Bradshaw, R. T.; Demerdash, O. N.; Dziedzic, J.; Mao, Y. Z.; Margul, D. T.; Swails, J.; Zeng, Q.; Case, D. A.; Eastman, P.; Wang, L. P.; Essex, J. W.; Head-Gordon, M.; Pande, V. S.; Ponder, J. W.; Shao, Y. H.; Skylaris, C. K.; Todorov, I. T.; Tuckerman, M. E.; Head-Gordon, T. Advanced Potential Energy Surfaces for Molecular Simulation. J. Phys. Chem. B 2016, 120 (37), 9811-9832.

(63) Lemkul, J. A.; Huang, J.; Roux, B.; MacKerell, A. D. An Empirical Polarizable Force Field Based on the Classical Drude Oscillator Model: Development History and Recent Applications. Chem. Rev. 2016, 116 (9), 4983-5013.

(64) Huang, J.; Simmonett, A. C.; Pickard, F. C.; MacKerell, A. D.; Brooks, B. R. Mapping the Drude polarizable force field onto a multipole and induced dipole model. J. Chem. Phys. 2017, 147 (16), 161702.

(65) Simmonett, A. C.; F, C. P., IV; Ponder, J. W.; Brooks, B. R. An empirical extrapolation scheme for efficient treatment of induced dipoles. J. Chem. Phys. 2016, 145 (16), 164101.

(66) Hadfield, A. T.; Harvey, D. J.; Archer, D. B.; MacKenzie, D. A.; Jeenes, D. J.; Radford, S. E.; Lowe, G.; Dobson, C. M.; Johnson, L. N. Crystal Structure of the Mutant D52S Hen Egg White Lysozyme with an Oligosaccharide Product. J. Mol. Biol. 1994, 243 (5), 856-872.

(67) Bottoni, A.; Miscione, G. P.; De Vivo, M. A theoretical DFT investigation of the lysozyme mechanism: Computational evidence for a covalent intermediate pathway. Proteins: Struct., Funct., Genet. 2005, 59 (1), 118-130.

(68) Farrer, R. A.; Fourkas, J. T. Orientational Dynamics of Liquids Confined in Nanoporous Sol-Gel Glasses Studied by Optical Kerr Effect Spectroscopy. Acc. Chem. Res. 2003, 36 (8), 605-612.

(69) Tortschanoff, A.; Portuondo Campa, E.; van Mourik, F.; Chergui, M. Optical Kerr effect studies of the dynamics of confined water. Microelectron. J. 2008, 39 (11), 1257-1258.

(70) Ratajska-Gadomska, B.; Bialkowski, B.; Gadomski, W.; Radzewicz, C. Ultrafast optical Kerr effect spectroscopy of water confined in nanopores of the gelatin gel. J. Chem. Phys. 2007, 126 (18), 184708

(71) Lamoureux, G.; Roux, B. Modeling induced polarization with classical Drude oscillators: Theory and molecular dynamics simulation algorithm. The. J. Chem. Phys. 2003, 119 (6), 3025-3039.

(72) Phillips, J. C.; Braun, R.; Wang, W.; Gumbart, J.; Tajkhorshid, E.; Villa, E.; Chipot, C.; Skeel, R. D.; Kale, L.; Schulten, K. Scalable 
molecular dynamics with NAMD. J. Comput. Chem. 2005, 26 (16), 1781-1802.

(73) Pandey, P.; Aytenfisu, A. H.; MacKerell, A. D.; Mallajosyula, S. S. Drude Polarizable Force Field Parametrization of Carboxylate and N-Acetyl Amine Carbohydrate Derivatives. J. Chem. Theory Comput. 2019, 15 (9), 4982-5000.

(74) Yu, H.; Whitfield, T. W.; Harder, E.; Lamoureux, G.; Vorobyov, I.; Anisimov, V. M.; MacKerell, A. D.; Roux, B. Simulating Monovalent and Divalent Ions in Aqueous Solution Using a Drude Polarizable Force Field. J. Chem. Theory Comput. 2010, 6 (3), 774786.

(75) Perticaroli, S.; Comez, L.; Paolantoni, M.; Sassi, P.; Lupi, L.; Fioretto, D.; Paciaroni, A.; Morresi, A. Broadband Depolarized Light Scattering Study of Diluted Protein Aqueous Solutions. J. Phys. Chem. B 2010, 114 (24), 8262-8269.

(76) Chu, J. W.; Trout, B. L.; Brooks, B. R. A super-linear minimization scheme for the nudged elastic band method. J. Chem. Phys. 2003, 119 (24), 12708-12717.

(77) Gonzalez, C.; Schlegel, H. B. An Improved Algorithm for Reaction-Path Following. J. Chem. Phys. 1989, 90 (4), 2154-2161.

(78) Jiang, W.; Hardy, D. J.; Phillips, J. C.; MacKerell, A. D.; Schulten, K.; Roux, B. High-Performance Scalable Molecular Dynamics Simulations of a Polarizable Force Field Based on Classical Drude Oscillators in NAMD. J. Phys. Chem. Lett. 2011, 2 (2), 87-92.

(79) Chowdhary, J.; Harder, E.; Lopes, P. E. M.; Huang, L.; MacKerell, A. D.; Roux, B. A Polarizable Force Field of Dipalmitoylphosphatidylcholine Based on the Classical Drude Model for Molecular Dynamics Simulations of Lipids. J. Phys. Chem. B 2013, 117 (31), 9142-9160.

(80) Smith, N. A.; Meech, S. R. Optically-heterodyne-detected optical Kerr effect (OHD-OKE): applications in condensed phase dynamics. Int. Rev. Phys. Chem. 2002, 21, 75-100.

(81) Geiger, L. C.; Ladanyi, B. M. Higher order interaction-induced effects on Rayleigh light scattering by molecular liquids. J. Chem. Phys. 1987, 87, 191.

(82) Ladanyi, B. M.; Liang, Y. Q. Interactioninduced contributions to polarizability anisotropy relaxation in polar liquids. J. Chem. Phys. 1995, 103, 6325 .

(83) Saito, S.; Ohmine, I. Third order nonlinear response of liquid water. J. Chem. Phys. 1997, 106, 4889.

(84) Lamoureux, G.; Harder, E.; Vorobyov, I. V.; Roux, B.; MacKerell, A. D. A polarizable model of water for molecular dynamics simulations of biomolecules. Chem. Phys. Lett. 2006, 418 (1), 245249.

(85) Blackman, R. B.; Tukey, J. W. The Measurement of Power Spectra, from the Point of View of Communications Engineering; Dover Publications, 1959.

(86) Frigo, M.; Johnson, S. G. The Design and Implementation of FFTW3. Proc. IEEE 2005, 93 (2), 216-231. 